\title{
Optical Control of the Rotational Angular Momentum of a Molecular Rydberg Wave Packet
}

\author{
R. S. Minns, R. Patel, J. R. R. Verlet, and H. H. Fielding* \\ Department of Chemistry, King's College London, Strand, London WC2R 2LS, United Kingdom
}

(Received 25 July 2003; published 10 December 2003)

\begin{abstract}
An intuitive scheme for controlling the rotational quantum state of a Rydberg molecule is demonstrated experimentally. We determine the accumulated phase difference between the various components of a molecular electron wave packet, and then employ a sequence of phase-locked optical pulses to selectively enhance or depopulate specific rotational states. The angular momentum composition of the resulting wave packet, and the efficiency of the control scheme, is determined by calculating the multipulse response of the time-dependent Rydberg populations.
\end{abstract}

DOI: 10.1103/PhysRevLett.91.243601

PACS numbers: $33.80 . \mathrm{Rv}$

Coherent control experiments in molecular systems have tended to employ complex waveforms [1-4] generated by feedback-controlled learning loops [5]. Despite the fact that they can be remarkably effective even in complex molecules [1-3,6] and biological systems [3], they are not intuitive and so it is difficult to unravel the underlying physics [6]. It is extremely tempting to try to control molecular processes from first principles and recognize the control mechanism. Ideal laboratories for the design of logical control schemes are Rydberg wave packets, since the phase parameters are readily determined. The relative phases of the interfering pathways are important since optical control processes rely on the interferences between different excitation pathways. Previous experiments controlling the dynamics of Rydberg electron wave packets have focused on simple atomic systems. Phase-shaped pulses have been employed to excite arbitrary wave packets [7] and to fully characterize their amplitude and phase profile [8-10]. Intuitive schemes exploiting trains of phase-locked optical pulses have been employed to create Schrödinger's cat states [11], to demonstrate Young's double slit interference in an atom [12], to control electronic orbital angular momentum [13], and the radial distribution [14]. In this Letter, we take a step further and develop an intuitive scheme to control the dynamics of a molecular system. We explore the dynamics of a molecular electron wave packet composed predominantly of two Rydberg series belonging to two different rotational quantum states of the molecular ion. We determine the accumulated phase difference and deduce an intuitive pulse sequence to obtain full control over the time-dependent populations of the rotational quantum state of the wave packet.

First, consider a wave packet composed of a superposition of two noninteracting Rydberg series, with different orbital angular momenta $l$ and quantum defects $\mu_{l}$, converging to the same ionization limit. Such a wave packet may be considered as being composed of two separate components and is written $\Psi(r, t)=\Sigma_{n l} a_{n l} \psi_{n l}(r) \times$ $\exp \left(-i \omega_{n l} t\right)$, where $\psi_{n l}(r)$ is the radial wave function of the eigenstate $|n l\rangle, \omega_{n l}=-1 / 2\left(n-\mu_{l}\right)^{2}$ is its frequency and $a_{n l}$ is its amplitude in the superposition. After an integer number of orbit periods $k$, each wave packet, corresponding to a channel $l$, accumulates a phase $2 \pi k \mu_{l}$, resulting in an accumulated phase difference between the two wave packets, $\phi_{l}=2 \pi k \Delta \mu_{l}$ [13]. The accumulated phase difference between two Rydberg series was recently exploited to control the orbital angular momentum composition of an atomic electron wave packet. In Xe, $\Delta \mu_{l}=$ 0.22 for the $n d[3 / 2]_{1}$ and $n s[3 / 2]_{1}$ Rydberg series lying below the lowest ionization limit. When $k=2$, i.e., after two orbit periods, $\phi_{l} \approx \pi$ and the phases of the paths coupling the two angular momentum components to the initial state are mismatched. If a second wave packet is launched at this time, it can be created so that it interferes constructively with the $s$ component of the evolved wave packet and destructively with the $d$ component, or vice versa. In this way, the orbital angular momentum character of the electron wave packet is controlled.

This Letter extends this idea, taking it from a simple atomic system to the more complex case of a molecule, where several degrees of freedom such as rotation and vibration are introduced. Rydberg molecules exhibit many of the dynamical complications found in large molecules, e.g., high density of rovibronic energy levels, nonadiabatic couplings, and various discrete-continuum interactions. Consider a wave packet composed of a superposition of two noninteracting molecular Rydberg series converging to different ionization limits corresponding to different rotational states of the molecular ion, which are separated by $\Delta E_{\text {rot }}$. This wave packet may be considered as consisting of two separate components each of which has its own electronic orbital angular momentum and is associated with a different rotational state of the molecular ion core. After a time $t$, the accumulated phase difference [15]

$$
\phi=2 \pi\left(\frac{\mu_{a}}{t_{\mathrm{cl}}^{a}}-\frac{\mu_{b}}{t_{\mathrm{cl}}^{b}}+\frac{1}{T_{\mathrm{rot}}}\right) t,
$$

where $a$ and $b$ label the Rydberg series corresponding to the lower and higher rotational quantum states of the molecular core, respectively, and $T_{\text {rot }}=2 \pi / \Delta E_{\text {rot }}$ is the 
rotational beat period. The accumulated phase for a given system now depends on energy as well as time. When both wave packets return to the core and overlap spatially (i.e., $t \approx t_{\mathrm{cl}}^{a} \approx t_{\mathrm{cl}}^{b}$, which is true for $\left.2 \nu_{a}^{2} \Delta E_{\mathrm{rot}} \ll 1\right)$ they will interfere with one another, and Eq. (1) can be rearranged to give the stroboscopic periods $T_{s}=T_{\text {rot }}(k+\Delta \mu)$ for $\phi=2 \pi k$ [15]. These interference effects have been observed in time-resolved spectra of molecular Rydberg wave packets in NO, manifesting themselves as plateaus in plots of the observed recurrence time as a function of the average principal quantum number in the superposition. If a second wave packet is launched at a time when the accumulated phase difference $\phi=\pi$, it can be engineered so that it interferes constructively with the Rydberg series associated with one rotational quantum state and destructively with the other. Removing one Rydberg series from the superposition has the effect of filtering out the rotational beat leaving pure electronic motion in the radial coordinate and the core in a welldefined rotational quantum state.

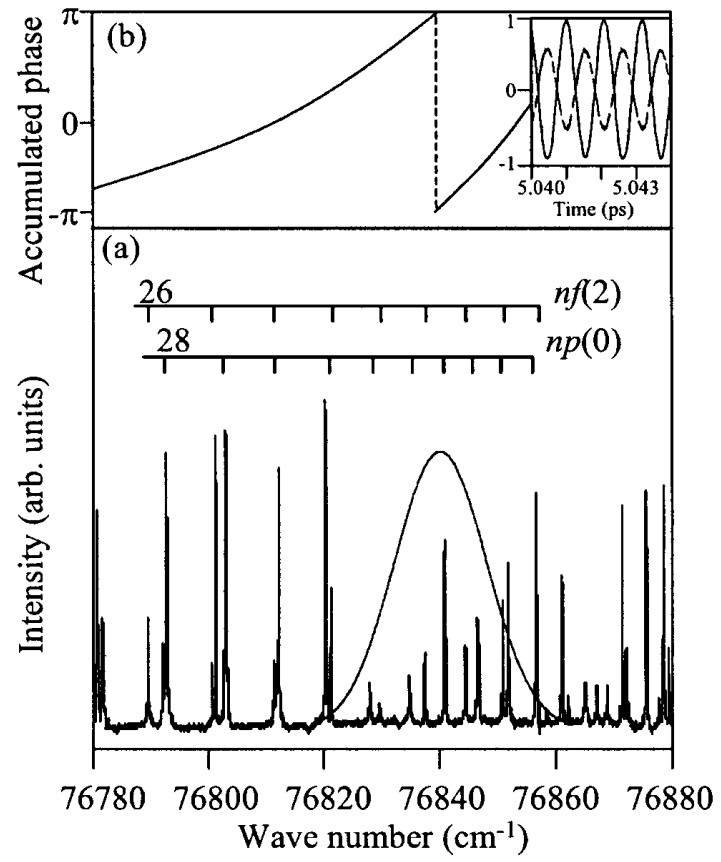

FIG. 1. (a) Part of the $1+1^{\prime}$ double resonance frequency spectrum of Rydberg states in $\mathrm{NO}$ excited via the $v^{\prime}=1, N^{\prime}=$ 0 level of the $\mathrm{A}^{2} \Sigma^{+}$state. Combs mark the positions of the dominant $n p(0)$ and $n f(2)$ series. A Gaussian centered at $\bar{n}_{0}=$ $33\left(\sigma=14 \mathrm{~cm}^{-1}\right)$ is superimposed on the spectrum, representing the optical pulse employed in our control experiments. (b) A plot of the accumulated phase difference $\phi$ at time $t_{\text {ave }}$, calculated using Eq. (1). Inset: The time-dependent interference term of the Rydberg population at time $t_{\text {ave }}$, calculated using Eq. (2) for excitation around $\bar{n}_{0}=33$. The contribution from the $n p(0)$ series is the solid (larger amplitude) line and that from the $n f(2)$ series is the dashed (smaller amplitude) line. It can be seen that the two components are phase mismatched around the time of the first recurrence.
We demonstrate our method using the NO molecule. The experimental setup has been described in detail elsewhere [16]. Briefly, we employ a resonantly enhanced one-nanosecond plus one-picosecond excitation scheme to create a wave packet that is composed predominantly of two Rydberg series converging to the $X^{1} \Sigma^{+}, v^{+}=1$ state of $\mathrm{NO}^{+}$. The Rydberg series are labeled $n p(0)$ and $n f(2)$ using the notation $n l\left(N^{+}\right)$where $N^{+}$is the rotational quantum number of the $\mathrm{NO}^{+}$core. For the experiments described here, a sequence of three identical phaselocked picosecond light pulses is required. The first two pulses are the excitation and control pulses, while the third is the detection pulse. The sequence of phase-locked pulses is generated in a three-armed Michelson interferometer which is feedback stabilized to within $0.01 \mathrm{fs}$ for periods of $>10 \mathrm{~h}$. The dynamics of the wave packet following just the excitation pulse, or the excitation plus control pulse sequence, are monitored using the optical Ramsey method [17]. This measures the autocorrelation function of the wave packet by allowing it to interact with another detection wave packet and observing interference fringes in the final Rydberg state population. The autocorrelation function is determined by calculating the root-mean-square of the amplitude of the fringes, which is measured by applying a delayed (100 ns) pulsed electric field $\left(1.7 \mathrm{kV} \mathrm{cm}^{-1}\right)$ to ionize the Rydberg state population and to accelerate the $\mathrm{NO}^{+}$ions along a $20 \mathrm{~cm}$ field-free flight tube towards a microchannel plate detector.

In Fig. 1(a) we present a high-resolution frequency spectrum of autoionizing Rydberg states in the range $27<n_{0}<48$, converging to the $v^{+}=1$ ionization limit of the $X^{1} \Sigma^{+}$state of $\mathrm{NO}^{+}$, which was recorded following excitation via the $A^{2} \Sigma^{+}\left(v^{\prime}=1, N^{\prime}=0, J^{\prime}=1 / 2\right)$ level of NO. It is clear that the $n p(0)$ and $n f(2)$ series dominate the autoionization spectrum. The accumulated phase difference $\phi$, between these two series at the average time of the first recurrence is calculated using Eq. (1) for the same region of the Rydberg spectrum and is plotted in modulo $2 \pi$ in Fig. 1(b). From this plot, it is apparent that $\phi$ is an odd multiple of $\pi$ at around $76840 \mathrm{~cm}^{-1}$, which corresponds to $\bar{n}_{0}=\left(-2 \varepsilon_{0}\right)^{-1 / 2}=33$, where $\bar{n}_{0}$ is a measure of the average energy below the $N^{+}=0$ ionization limit. The effect of $\phi$ on the optically induced interference fringes in a wave packet spectrum can be determined by separating out the $n p(0)$ and $n f(2)$ series and using the experimental excitation energies $\omega$ and intensities $a_{N^{+}}^{2}(\omega)$. The subscript $N^{+}$is used to label the rotational quantum state of the molecular ion core. The spectrum is convoluted with a Gaussian of full-width at halfmaximum $\sigma=14 \mathrm{~cm}^{-1}$, which is equivalent to our experimental laser pulse, centered around $\bar{n}_{0}=33$. The time-dependent interference term of the Rydberg population $A_{N^{+}}^{2}(t)$ for each rotational state of the molecular ion core is calculated around the average time of the first recurrence, $t=t_{\mathrm{cl}}$, using 


$$
A_{N^{+}}^{2}(t)=\operatorname{Re} \sum_{\omega} a_{N^{+}}^{2}(\omega) e^{-4 \ln 2\left(\omega-\omega_{L}\right)^{2} / \sigma^{2}} e^{-i \omega t},
$$

where $\omega_{L}$ is the average excitation frequency of the laser pulse. The interference terms $A_{0}^{2}(t)$ and $A_{2}^{2}(t)$, are plotted in the inset to Fig. 1(b) as solid (larger amplitude) and dashed (smaller amplitude) lines, respectively, around the time of the average classical orbit period $t_{\text {ave }}=5.04$ ps. The interference terms are out of phase with one another. It is clear that adding a second wave packet at $t_{2}=$ $5.041 \mathrm{ps}$ would result in constructive interference with the $N^{+}=0$ series and destructive interference with the $N^{+}=2$ series, leaving a molecular electron wave packet in a well-defined rotational quantum state with $N^{+}=0$. Flipping the optical phase of the second laser pulse by $\pi$ will reverse the situation and leave the molecular electron wave packet in a well-defined rotational quantum state with $N^{+}=2$.

This control scheme is illustrated in Fig. 2 where (a) are experimental spectra and (b) are the corresponding calculated spectra. The bottom trace in Fig. 2(a) shows the experimental recurrence spectrum following excitation of a single wave packet. The first recurrence exhibits the double peak structure that has been observed in previous experiments [15]. The dip corresponds to destructive interference between the two wave packets when they return to the core with an accumulated phase difference of $\pi$, and is observed at time $t_{\text {ave }}$. Adding the second control wave packet to the system at this time allows

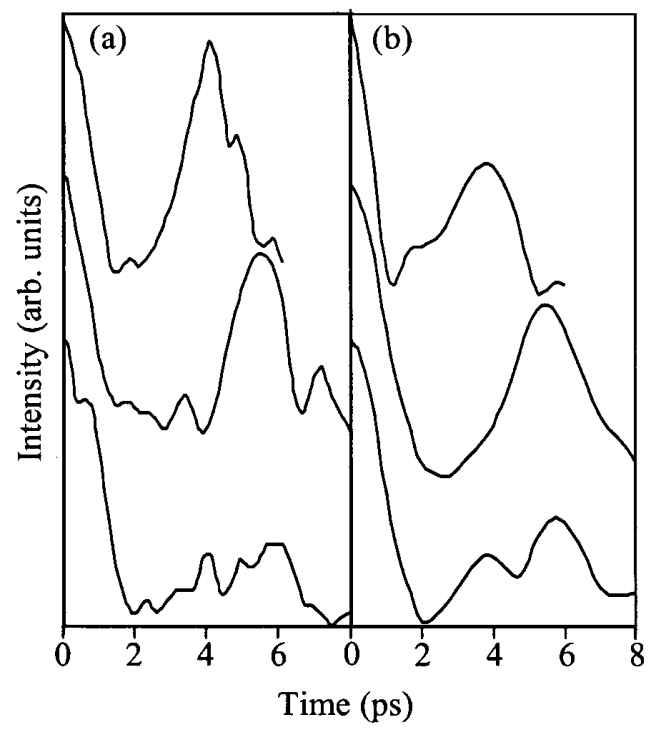

FIG. 2. Experimental (a) and calculated (b) recurrence spectra of a wave packet with $\bar{n}_{0}=33$. The bottom spectrum is that of a single wave packet. The middle spectrum is that of a sequence of pulses separated by $t_{\text {ave }}$ with a suitable phase difference to leave the $N^{+}=0$ series only. The top spectrum is that of a sequence of pulses separated by $t_{\text {ave }}$ with a suitable phase difference to leave the $N^{+}=2$ series only. Note that all spectra, experimental and calculated, are plotted on the same vertical scale, normalized on the peak at $t=0$. us to manipulate the final state Rydberg population. Experimental recurrence spectra are recorded following excitation of two pulses separated by $t_{\text {ave }}$ with a suitable phase added to leave the $N^{+}=0$ series only (middle trace) or the $N^{+}=2$ series only (top trace). For a given excitation energy the period of motion of a wave packet in a system with the core in the lower rotational state will be longer and this is illustrated well by these spectra. The position of the first recurrence in the middle plot is $5.6 \mathrm{ps}$, which agrees well with the calculated orbit period of a wave packet with $\bar{n}_{0}=33, t_{\mathrm{cl}}=2 \pi \bar{n}^{3}=5.5 \mathrm{ps}$. The first recurrence in the upper plot appears at 4.3 ps which is close to the calculated orbit period for $\bar{n}_{2}=31.2, t_{\mathrm{cl}}=$ $4.6 \mathrm{ps}$. Effectively, the double peak feature has been deconvoluted into its two contributing components.

The equivalent calculated recurrence spectra are obtained using the modified autocorrelation function

$$
\begin{aligned}
\langle\Psi(0) \mid \Psi(t)\rangle=\mid \sum_{N^{+}, \omega} & a_{N^{+}}^{2}(\omega) e^{-4 \ln 2\left(\omega-\omega_{L}\right)^{2} / \sigma^{2}} \\
& \times\left[e^{-i \omega t}+e^{-i \omega\left(t+t_{2}\right)}\right] .
\end{aligned}
$$

The first term in square brackets describes the temporal evolution of the excitation wave packet created at $t=0$ and the second term describes the evolution of the control wave packet launched at $t_{2}=t_{\text {ave }}+t_{\phi}$, where $t_{\phi}$ is the delay which corresponds to an optical phase $\phi$. All spectra are normalized on the $t=0$ peak and plotted on the same vertical scale and, in the experiments involving a sequence of pulses, the zero of time corresponds to the launch of the control wave packet. We can investigate the effectiveness of the control scheme in more detail by calculating the population distribution after a sequence of two pulses $A^{2}(\omega)$, assuming the intensity of the laser pulses is sufficiently weak that the population of the intermediate $A$ state remains virtually unchanged. The method is the same as that employed to determine the population distribution in Schrödinger cat states [18]:

$$
\begin{aligned}
A^{2}(\omega) \propto\left[\sum_{N^{+}}\right. & a_{N^{+}}(\omega) e^{-2 \ln 2\left(\omega-\omega_{L}\right)^{2} / \sigma^{2}} \\
& \left.\times\left[1+e^{i \Delta t_{2}} e^{i\left(\omega-\omega_{L}\right) t_{2}}\right]\right]^{2} .
\end{aligned}
$$

$\Delta=\omega_{L}-\omega_{0}$ where $\omega_{0}$ is the frequency of the intermediate. After launching the second control pulse at $t_{2}=5.041 \mathrm{ps}$ [corresponding to the middle trace in Fig. 2(b)] the population distribution is calculated using Eq. (4) and plotted as the positive trace in Fig. 3. The peaks correspond to the line positions of the $n p(0)$ Rydberg series and it is clear that there is very little contamination from other states in the wave packet, thus verifying that the molecular ion core is in a welldefined quantum state with $N^{+}=0$. Flipping the phase of the optical pulse by $\pi$ generates the population 


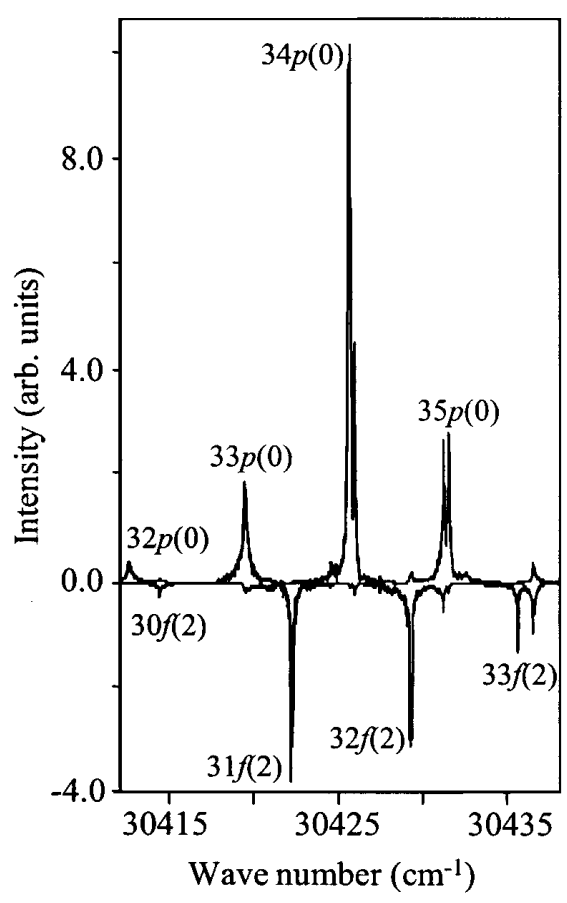

FIG. 3. Population distributions determined using Eq. (4). Positive peaks represent the final state distribution obtained when the second pulse is added at $t_{2}=5.041 \mathrm{ps}$ [corresponding to the middle trace in Fig. 2(b)]. Flipping the phase of the second wave packet yields the population distribution that is plotted on a negative scale.

distribution plotted as the negative trace. The peaks in this plot correspond to the line positions of the $n f(2)$ Rydberg series, and again there is very little contamination from other states. In this case the molecular ion core has a well-defined quantum state with $N^{+}=2$. It is clear from these two plots that the level of control is excellent.

In summary, we have demonstrated control over the molecular motion of a Rydberg system. Using a train of phase-locked pulses we have been able to select the rotational quantum state of the molecular ion core. The control scheme is not specific to the quantum numbers demonstrated here but can be extended to any rotational state of the core by simply exciting via a different intermediate. The degree of control has been fully characterized by determining the population distribution after a particular pulse sequence and it is extremely effective. The additional phase parameters inherent in a molecular system aid the control mechanism in some respects, as one can search for a phase mismatch specifically at the first recurrence where dispersion is less of an issue. In general terms, if the accumulated phase difference can be determined, a logical pulse sequence can be designed to control the final state quantum distribution. The next step will be to look at a system in which the Rydberg series are coupled. By improving our understanding of the link between optical phase and molecular and electronic phase in this stepwise way, we are one step nearer designing intuitive control schemes in complex molecular systems with more degrees of freedom.

We thank the EPSRC for equipment funding and for financial support for R. S. M., R. P., and H. H. F. We also thank King's College London for financial support (J. R. R. V.). We thank J. Hauer, R. A. L. Smith, and V. G. Stavros for experimental assistance. We are grateful to the Rutherford Appleton laser loan pool for allowing us the use of the Continuum $8000 \mathrm{Nd}: Y A G$ laser and the Sirah Precisionscan dye laser.

*Present address: Department of Chemistry, University College London, Christopher Ingold Laboratories, 20 Gordon Street, London, U.K. WC1H 0AJ.

[1] A. Assion, T. Baumert, M. Bergt, T. Brixner, B. Kiefer, V. Seyfried, M. Strehle, and G. Gerber, Science 282, 919 (1998).

[2] M. Bergt, T. Brixner, B. Kiefer, M. Strehle, and G. Gerber, J. Phys. Chem. A 103, 10381 (1999).

[3] J. L. Herek, W. Wohlleben, R. J. Cogdell, D. Zeidler, and M. Motzkus, Nature (London) 417, 533 (2002).

[4] E. Hertz, G. Nersisyan, N. A. Papadogiannis, and D. Charalambidis, J. Chem. Phys. 118, 595 (2003).

[5] R. S. Judson and H. Rabitz, Phys. Rev. Lett. 68, 1500 (1992).

[6] C. Daniel, J. Full, L. Gonzalez, C. Lupulescu, J. Manz, A. Merli, S. Vajda, and L. Woste, Science 299, 536 (2003).

[7] T.C. Weinacht, J. Ahn, and P. H. Bucksbaum, Nature (London) 397, 233 (1999).

[8] T. C. Weinacht, J. Ahn, and P. H. Bucksbaum, Phys. Rev. Lett. 80, 5508 (1998).

[9] T. C. Weinacht, J. Ahn, and P. H. Bucksbaum, Phys. Rev. Lett. 81, 3050 (1998).

[10] J. Ahn, T. C. Weinacht, and P. H. Bucksbaum, Science 287, 463 (2000).

[11] M.W. Noel and C. R. Stroud, Phys. Rev. Lett. 77, 1913 (1996).

[12] M.W. Noel and C. R. Stroud, Phys. Rev. Lett. 75, 1252 (1995).

[13] J. R. R. Verlet, V. G. Stavros, R. S. Minns, and H. H. Fielding, Phys. Rev. Lett. 89, 263004 (2002).

[14] J. R. R. Verlet, V. G. Stavros, R. S. Minns, and H. H. Fielding, J. Phys. B 36, 3683 (2003).

[15] R. A. L. Smith, V. G. Stavros, J. R. R. Verlet, H. H. Fielding, D. Townsend, and T. P. Softley, J. Chem. Phys. 119, 3085 (2003).

[16] R. A. L. Smith, J. R. R. Verlet, and H. H. Fielding, Phys. Chem. Chem. Phys. 5, 3567 (2003).

[17] L. D. Noordam, D. I. Duncan, and T. F. Gallagher, Phys. Rev. A 45, 4734 (1992).

[18] X. Chen and J. A. Yeazell, Phys. Rev. A 55, 3264 (1997). 